\title{
CONGRUENCE AND SUPERPOSABIIITY IN ELLIPTIC SPACE
}

\author{
BY \\ LEONARD M. BLUMENTHAL
}

1. Introduction. Two subsets $A, B$ of a metric space $M$ are congruent provided a mapping $f(A)=B$ exists such that $p, q \in A$ implies $p q=\operatorname{dist.~}(p, q)$ =dist. $(f(p), f(q))=f(p) f(q)$. Congruence of $A$ and $B$ is denoted by $A \approx B$ or by $A \approx{ }_{f} B$ if one wishes to indicate a particular mapping $f$ which transforms $A$ congruently onto $B$.

Two subsets of a metric space $M$ are superposable provided a congruent mapping of $M$ onto itself exists which maps one of the subsets onto the other. It follows that two subsets $A, B$ are superposable if and only if a congruence $f$ exists between the sets which can be extended to the whole space. It should be observed, however, that superposability of two sets does not imply that each congruent mapping of one onto the other can be extended to the whole space. Thus, for example, if $M$ is the line segment seg. $\left(p, q^{\prime}\right)$, with middle-point labelled both $p^{\prime}$ and $q$, then the two pairs of points $(p, q),\left(p^{\prime}, q^{\prime}\right)$ are superposable since the congruent mapping $f(p)=q^{\prime}, f(q)=p^{\prime}$ can be extended to the segment by a reflection in $q$, but the congruence $g(p)=p^{\prime}, g(q)=q^{\prime}$ can obviously not be extended to the space. Moreover, a given congruence between two subsets of a metric space does not imply either that that congruence or any other between the subsets can be extended to a congruence of the whole space with itself.

It is well known that euclidean, spherical, and hyperbolic spaces possess the strong property (a) any two congruent subsets are superposable, and the even stronger property (b) any congruence between any two subsets can be extended to a congruence of the whole space with itself. In a space with property (b) each two congruent subsets may be called freely supelposable, and (as G. Birkhoff [1](1) has recently shown) among all metric spaces in which each two points are joined by a segment, locally unique, only in the three spaces named above are congruent subsets freely superposable.

In this paper congruence and superposability in $\boldsymbol{n}$-dimensional elliptic space $E_{n, r}$ are studied. Since congruent but not superposable subsets exist in every elliptic space of dimension greater than $1(\$ 3)$ the $E_{n, r}(n>1)$ does not have property (a). Moreover, it will be seen ( $\$ 6)$ that not every congruence between superposable subsets of $E_{n, r}$ is necessarily extendible to the whole space. These circumstances give rise to two problems: (1) to find neces-

Presented to the Society, April 27, 1946, and November 29, 1946; received by the editors November 25, 1946.

(1) Numbers within brackets refer to the bibliography concluding this paper. 
sary and sufficient conditions for the superposability of two congruent subsets of $E_{n, r}$ and (2) to determine when a given congruence $f$ between two subsets of $E_{n, r}$ may be extended to the whole space.

Solutions of these problems are given in $\$ 4$ (finite subsets) and $\$ 5$ (arbitrary subsets). A principal result (Theorem 5.4) is that a given congruence between two subsets $P, Q$ of $E_{n, r}$ can be extended to the whole space if and only if each of the sub-congruences between corresponding $(n+1)$-tuples of $P$ and $Q$ can be so extended. This leads to the introduction of a new concept "superposability order" (analogous to the notion of congruence order) which gives rise to the consideration and characterization of so-called pseudo superposable pairs of sets $(\S 6)$.

The writer is not aware of any previous investigation of the matters treated in this paper. The problems dealt with here do not arise in those special spaces (euclidean, spherical, hyperbolic) which are most studied, since in them congruence and superposability are logically equivalent notions; while only recently has elliptic space been studied from that point of view (the metric) which seems to furnish the most natural means for their formulations and solutions [2].

In conclusion it is pointed out that since nonsuperposable congruent subsets of $E_{n, r}$ exist, superposability is not a congruence invariant and hence a purely metric characterization of superposable subsets is not possible. Congruence is, of course, a necessary condition for superposability, but in elliptic space the relationship is inextricably bound up with the position of the sets in the space. In view of this fact, it is of interest co note how the positional feature enters into the "almost metric" conditions for superposability that we obtain.

2. Some definitions and notations. A convenient definition (model) of the $n$-dimensional elliptic space $E_{n, r}$ of space constant $r$ is obtained by identifying diametrically opposite points of the metrically convex (geodesically metrized) $n$-dimensional boundary of the sphere of radius $r$ in euclidean space of $n+1$ dimensions. Denoting this convex $n$-dimensional spherical space by $S_{n, r}$, then $E_{n, r}$ is the metric transform $\left({ }^{2}\right)$ of $S_{n, r}$ by the function

$$
\begin{array}{lll}
F(x)=x, & x=p q, & p q \leqq \pi r / 2, \\
F(x)=\pi r-x, & x=p q, & p q>\pi r / 2,
\end{array}
$$

where $p, q \in S_{n, r}$ and $p q$ denotes distances in $S_{n, r}$. It is convenient to write $E_{n, r}=F\left(S_{n, r}\right)$ and to refer to $F$ as the $\pi r$-supplementation function.

Thus if distance on the convex spherical space $S_{n, r}$ is redefined so that each spherical distance not exceeding $\pi r / 2$ is left unaltered, while each spherical distance that exceeds $\pi r / 2$ is replaced by its supplement, the $n$-dimensional elliptic space $E_{n, r}$ results. It is a compact metric space of diameter $\pi r / 2$.

(2) See [3, pp. 334-335]. 
The notations $A \sim_{f} B, A \approx \approx_{f} B, A \cong{ }_{f} B$ denote one-to-one correspondence, congruence, and superposability, respectively, of the sets $A, B$, established by the function $f$. If two $m$-tuples $p_{1}, p_{2}, \cdots, p_{m}$ and $q_{1}, q_{2}, \cdots, q_{m}$ are related by one of these relations as, for example,

$$
p_{1}, p_{2}, \cdots, p_{m} \approx q_{1}, q_{2}, \cdots, q_{m},
$$

it is understood that $p_{i}$ and $q_{i}$ correspond (by $f$ if the function forms part of the symbol of congruence) for each index $i=1,2, \cdots, m$. When it is desired to state merely that two $m$-tuples are congruent or superposable (without indicating pairs of corresponding points), the sets are inclosed in parentheses; for example,

$$
\left(p_{1}, p_{2}, \cdots, p_{m}\right) \cong\left(q_{1}, q_{2}, \cdots, q_{m}\right),
$$

where the parentheses about the first $m$-tuple might be omitted.

A congruent mapping of a space onto itself is called a motion.

Let $P, Q$ be two subsets of $E_{n, r}$ with $P \sim_{f} Q$. Two corresponding subsets $A_{P}, B_{Q}$ of $P$ and $Q$, respectively, are said to be $f$-superposable $\left(A_{P} \cong_{f} B_{Q}\right)$ provided there exists a motion $\Gamma$ such that $A_{P} \sim_{f} B_{Q}$ is identical with $A_{P} \sim_{\Gamma} B_{Q}$.

If an $m$-tuple of $E_{n, r}$ is ordered so that no one of its points has distance $\pi r / 2$ from each preceding (succeeding) point, the $m$-tuple is in left-hand (right-hand) apolar order.

3. Non-superposable congruent subsets. It has been remarked that the elliptic line $E_{1, r}$ contains three points $p_{1}, p_{2}, p_{3}$ with $p_{i} p_{j}=\pi r / 3(i, j=1,2,3)$ which are congruent with three points of $E_{2, r}$ which do not lie on any $E_{1, r}\left({ }^{3}\right)$. In this section it is shown that for every integer $k$ greater than 1 , the $E_{k, r}$ contain subsets of arbitrarily high power (not exceeding $c$ ) which are congruent with elliptic subsets which do not lie in any $E_{k, r}$, while, on the other hand, a subset of $E_{1, r}$ which is congruent with an elliptic set not contained in a line must consist of exactly three points. I't is seen, further, how nonsuperposable congruent subsets, each of which is contained irreducibly in the same dimensional elliptic space, may be constructed, of arbitrary power not exceeding $\mathrm{c}$.

Lemma 3.1. The elliptic line $E_{1, r}$ is congruent with the convex circle $S_{1, r / 2}$.

Proof. Let $E_{1, r}$ be obtained by identifying diametral pointpairs of the convex circle $S_{1, r}$ and construct $S_{1, r / 2}$ tangent internally to $S_{1, r}$. Then the center $O$ of $S_{1, r}$ is the point of contact of $S_{1, r / 2}$ with the diameter of $S_{1, r}$ tangent to it, and a one-to-one correspondence between the points of $E_{1, r}$ and $S_{1, r / 2}$ is obtained by associating with a point $p$ of the elliptic line that point $p^{\prime}$ of the circle $S_{1, r / 2}$ in which it is intersected by the straight line $o p$, where $p^{\prime}$ is chosen distinct from $o$ except when the straight line $o p$ is the tangent line to $S_{1, r / 2}$. If $p, q$ and $p^{\prime}, q^{\prime}$ are points of $E_{1, r}, S_{1, r / 2}$, respectively,

(3) [2, p. 382]. See also H. Busemann, Metric methods in Finsler spaces and in the foundations of geometry, Princeton University Press, 1942, p. 186. 
which correspond by this association, then

$$
p q=r \cdot(\Varangle p o q)=r \cdot\left(\Varangle p^{\prime} o q^{\prime}\right)=r\left(1 / 2 \Varangle p^{\prime} o^{\prime} q^{\prime}\right)=p^{\prime} q^{\prime},
$$

and the lemma follows( $($ ).

LEMMA 3.2. If $p_{1}, p_{2}, p_{3}, p_{4}$ are four points of $E_{1, r}$ then at least two of the four triples they contain are linear $\left(^{5}\right)$.

Proof. This follows at once from the preceding lemma and the fact that four points of a convex circle have this property.

THEOREM 3.1. If $p_{1}, p_{2}, \cdots, p_{m}$ are $m$ points of $E_{n, r}$ congruent with $p_{1}^{\prime}, p_{2}^{\prime}, \cdots, p_{m}^{\prime}$ of $E_{1, r}(m>3)$, then $p_{1}, p_{2}, \cdots, p_{m}$ lie on an elliptic line.

Proof. It follows from Lemma 3.2 that for every $i, j$ the points $p_{1}, p_{2}$, $p_{i}, p_{i}(i, j=3,4, \cdots, m)$ contain two linear triples. Since a linear triple of $E_{n, r}$, necessarily lies on a line $E_{1, r}$, and two distinct lines of $E_{n, r}$ have exactly one point in common, then $p_{1}, p_{2}, p_{i}, p_{j}$ lie on $E_{1, r}\left(p_{1}, p_{2}\right)(i, j=3,4, \cdots, m)$, and the theorem is proved $\left({ }^{6}\right)$.

Thus if a subset of $E_{1, r}$ is congruent to an elliptic set which is not on any $E_{1, r}$ then the subset consists of exactly three points. It is easy to show that the class of such triples of $E_{1, r}$ is characterized by the following property: each triple of the class has diameter less than $\pi r / 2$ and the sum of the three distances equals $\pi r$.

Turning now to the plane $E_{2, r}$ it is clear that subsets exist (for example, the equilateral quadruple with edge $r \cdot \operatorname{arc} \cos (1 / 3)$ ) which are congruent with four points of $E_{3, r}$ not lying in any $E_{2, r}$. But in view of Theorem 3.1 it might be conjectured that such planar sets consist of exactly four point or, failing this, that the number of points such sets can possess is less than a fixed integer $m$. That conjectures of this nature are false is shown in the following theorem:

TheOREM 3.2. For every cardinal number $m(3<m \leqq c)$ the elliptic plane contains a subset of power $m$ which is congruent with an elliptic subset not con-

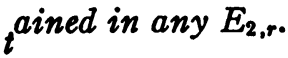

Proof. An example of such a planar subset has just been given for $m=4$. Consider now the planar quintuple $p_{1}, p_{2}, \cdots, p_{5}$ with $p_{1}, p_{2}, p_{3}$ on a line $E_{1, r}, p_{i} p_{j}=\pi r / 3(i, j=1,2,3), p_{4}$ and $p_{5}$ reflections of each other in this

(4) $\Varangle p o q\left(=\Varangle p^{\prime} \circ q^{\prime}\right)$ denotes the radian measure of the smaller of the two angles made by the straight lines $o p$ and $o q$, while $\Varangle p^{\prime} o^{\prime} q^{\prime}$ is the radian measure (not exceeding $\pi$ ) of the central angle obtained by joining $p^{\prime}, q^{\prime}$ to the center $o^{\prime}$ of $S_{1, r / 2}$.

(๖) A triple of points is linear provided the sum of two of the three distances determined equals the third.

( $)$ It is noted that while a linear triple of $E_{n, r}$ always lies on a line $E_{1, r}$, three points of $E_{n, r}$ congruent with three points of $E_{1, r}$ do not necessarily lie on an $E_{1, r .}$ 
line, with $p_{1} p_{4}=p_{2} p_{4}=p_{1} p_{5}=p_{2} p_{5}=\pi r / 3$. Then $p_{3} p_{4}=p_{3} p_{5}=\pi r / 2$ and $p_{4} p_{5}=$ $r \cdot \arccos (1 / 3)$.

If we rotate the elliptic plane about the line $E_{1, r}\left(p_{1}, p_{2}, p_{8}\right)$ into the elliptic three-space $E_{3, r}$, it follows from the continuity of the metric that a point $p_{5}^{\prime}$ of $E_{3, r}$ exists such that $p_{5}^{\prime}$ is not an element of $E_{2, r}\left(p_{1}, p_{2}, \cdots, p_{5}\right)$ and $p_{1}, p_{2}, p_{3}, p_{4}, p_{5} \approx p_{1}, p_{2}, p_{3}, p_{4}, p_{5}^{\prime}$.

Since an elliptic plane is uniquely determined by three points not on an elliptic line, the points $p_{1}, p_{2}, p_{3}, p_{4}, p_{6}^{\prime}$ do not lie on a plane, for if they did that plane would be identical with the plane containing $p_{1}, p_{2}, \cdots, p_{5}$ since the two planes would have the non- $E_{1, r}$ triple $p_{1}, p_{2}, p_{4}$ in common. This is not possible since $p_{5}^{\prime}$ does not lie in $E_{2, r}\left(p_{1}, p_{2}, \cdots, p_{5}\right)$.

It is clear that by adjoining to the quintuple $p_{1}, p_{2}, \cdots, p_{5}$ points of the line $E_{1, r}\left(p_{1}, p_{2}, p_{3}\right)$ a planar set of arbitrary power (not exceeding c) may be obtained which is congruent with a subset of $E_{8, r}$ not contained in any elliptic plane.

There is no difficulty in extending this example. Thus, if we select five points $p_{1}, p_{2}, \cdots, p_{6}$ of $E_{2, r}$, not lying in a line, a sixth point $p_{6}$ not in this $E_{2, r}$ determines with it a unique $E_{3, r}$. If $p_{7}$ denotes the reflection of $p_{6}$ in $E_{2, r}\left(p_{1}, p_{2}, \cdots, p_{8}\right)$ and dist. $\left(p_{6}, E_{2, r}\left(p_{1}, p_{2}, \cdots, p_{8}\right)\right)$ is not too small, rotation of $E_{3, r}$ about this plane into the $E_{4, r}$ yields a point $p_{7}^{\prime}$ such that $p_{1}, p_{2}, \cdots, p_{7} \approx p_{1}, p_{2}, \cdots, p_{7}^{\prime}$, with $p_{1}, p_{2}, \cdots, p_{6}, p_{7}^{\prime}$ not contained in any $E_{8, r}$. In this manner the following result is obtained:

THEOREM 3.3. For every integer $k>1$ and every cardinal number $m(k+1<m \leqq c)$ each $k$-dimensional elliptic space $E_{k, r}$ contains a subset of power $m$ which is congruent with a subset of $E_{n, r}$ ( $n$ finite) which is not contained in any $k$-dimensional elliptic space $E_{k, r}$.

COROLlaRY. For $k>1$ there is no purely metric means of distinguishing subsets of one elliptic space from certain subsets contained irreducibly in an elliptic space of different dimension.

It follows that in elliptic space the property of being contained in a subspace of given dimension is not a congruence invariant. An $m$-tuple which is "dependent" in the classical sense (that is, contained in a subspace of dimension less than $m-1$ ) may be congruent with an "independent" $m$-tuple.

Since any congruent transformation of $E_{n, r}$ onto itself evidently transforms $k$-dimensional subspaces onto $k$-dimensional subspaces, it is clear that two congruent subsets which lie irreducibly in subspaces of different dimensions are not superposable. Hence elliptic space of dimension greater than 1 contains congruent subsets of arbitrary power (not exceeding $c$ ) such that no congruence between the sets can be extended to the whole space.

But the phenomenon of congruent non-superposable sets is not restricted to sets which are contained irreducibly in subspaces of different dimensions. The elliptic plane contains points $p_{1}, p_{2}, p_{8}, p_{4}, p_{4}^{\prime}$ such that $p_{1} p_{2}=\pi r / 3$, 
$p_{1} p_{4}=p_{2} p_{3}=\pi r / 6, \quad p_{1} p_{3}=r \cdot \operatorname{arc} \cos 3^{1 / 2} / 6, \quad p_{2} p_{4}=r \cdot \operatorname{arc} \cos 3^{1 / 2} / 10, \quad p_{3} p_{4}$ $=r \cdot \operatorname{arc} \cos 2^{3 / 2} / 15$, and $p_{1} p_{4}^{\prime}=p_{1} p_{4}, p_{2} p_{4}^{\prime}=p_{2} p_{4}$, with spher. dist. $\left(p_{3}, p_{4}\right)$ $\neq$ spher. dist. $\left(p_{3}, p_{4}^{\prime}\right)=r \cdot \operatorname{arc} \cos \left(-2^{3 / 2} / 15\right)$.

The points $p_{1}, p_{2}, p_{3}$ are on the locus of points of $E_{2, r}$ equidistant from $p_{4}$ and $p_{4}^{\prime}$ (that is, they lie on two mutually perpendicular elliptic lines) but they are not on any $E_{1, r}$. The two quadruples $p_{1}, p_{2}, p_{3}, p_{4}$ and $p_{1}, p_{2}, p_{3}, p_{4}^{\prime}$ are congruent planar quadruples, but they are not superposable, for any congruent transformation of $E_{2, r}$ onto itself which mapped one quadruple onto the other would leave the three lines $E_{1, r}\left(p_{i}, p_{j}\right)(i, j=1,2,3 ; i \neq j)$ pointwise invariant (since $\left.p_{i} p_{j} \neq \pi r / 2(i, j=1,2,3)\right)$. Since the lines are pairwise distinct, this implies that the plane itself is left pointwise invariant, which is impossible.

By adjoining points of $E_{1, r}\left(p_{1}, p_{2}\right)$ it is clear how two congruent sets of arbitrary power (not exceeding $c$ ) may be constructed which are not superposable, and each of which is contained irreducibly in the elliptic plane. Similar examples are easily found in each $E_{k, r}(k>1)$.

It is observed that the triples $p_{1}, p_{2}, p_{3}$ and $p_{1}, p_{2}, p_{4}$ are obviously each superposable with the two corresponding triples of $p_{1}, p_{2}, p_{3}, p_{4}^{\prime}$, and examples of congruent but nonsuperposable quadruples of $E_{2, r}$ may be found with three pairs of corresponding triples superposable. If, however, two congruent planar quadruples have their four pairs of corresponding triples superposable, then the quadruples are superposable (Theorem 5.4).

In concluding this section we note an application of Theorem 3.1 to quadratic form theory.

Theorem 3.4. Let $Q$ denote the quadratic form

$$
\sum_{i=1}^{m} x_{i}^{2}+2 \sum_{i<j=1}^{m} \epsilon_{i j} a_{i j} x_{i} x_{j}, \quad \epsilon_{i j}= \pm 1 ; 0 \leqq a_{i j}<1
$$

$(i, j=1,2, \cdots, m ; i<j, m>3)$. If each sub-form in four variables obtained from $Q$ by setting equal to zero all but four of the $m$ variables can be given rank 2 by properly choosing the epsilons appearing in it, then (1) $Q$ can be given rank 2 by a proper selection of $\epsilon_{i j}(i, j=1,2, \cdots, m)$ and (2) every positive semi-definite form $Q^{\prime}$ obtained from $Q$ by a choice of the epsilons has rank 2.

Proof. If we introduce a semimetric $m$-tuple $p_{1}, p_{2}, \cdots, p_{m}$ with $p_{i} p_{j}$ $=r \cdot \operatorname{arc} \cos a_{i j}(i, j=1,2, \cdots, m ; i<j), r>0$, the hypothesis implies that each four of these $m$ points is congruently contained in an elliptic line $E_{1, r}$, and hence the whole $m$-tuple is also. Thus $p_{1}, p_{2}, \cdots, p_{m}$, $\approx p_{1}^{\prime}, p_{2}^{\prime}, \cdots, p_{m}^{\prime}$, with the "primed" points in $E_{1, r}$, and the first conclusion of the theorem is insured by the elliptic imbedding theorem $\left({ }^{7}\right)$.

If, now, $Q^{\prime}$ is any positive semi-definite quadratic form obtained from $Q$

(ग) See [3, p. 336]. 
by the process described in the theorem, it follows from the same imbedding theorem that $p_{1}, p_{2}, \cdots, p_{m} \approx p_{1}^{\prime \prime}, p_{2}^{\prime \prime}, \cdots, p_{m}^{\prime \prime}$, with the latter points in an elliptic space $E_{k, r}$, where $k+1$ is the rank of $Q^{\prime}$. But then $p_{1}^{\prime}, p_{2}^{\prime}, \cdots, p_{m}^{\prime}$ $\approx p_{1}^{\prime \prime}, p_{2}^{\prime \prime}, \cdots, p_{m}^{\prime \prime}$, and since $m>3$ the latter $m$-tuple must, according to Theorem 3.1, lie in an elliptic line $E_{1, r}$; that is, the rank of $Q^{\prime}$ is 2 .

Remark 3.1. Since the form $x_{1}^{2}+x_{2}^{2}+x_{3}^{2}+x_{1} x_{2}+x_{1} x_{3}-x_{2} x_{3}$ is positive semidefinite of rank 2, while the form obtained from it by changing the sign of the last term is positive definite (rank 3 ) the analogue of Theorem 3.4 for forms in three variables is not valid.

Remark 3.2. Theorem 3.3 precludes the possibility of extending Theorem 3.4 in the obvious manner (that is, by replacing "four" by " $k+2$ " and "rank 2 " by "rank $k$ " $(k>2)$ ) even if $m$ be taken arbitrarily large.

Remark 3.3. Using the superposability criteria to be developed in the next section (Theorem 4.1), together with the fact that since $E_{1, r} \approx S_{1, r / 2}$ any congruence between two subsets of $E_{1, r}$ can be extended to the whole space, Theorem 3.4 can be greatly strengthened by adding a third conclusion (which makes the second conclusion obvious); namely (3) the only choices of the epsilons which make $Q^{\prime}$ positive semi-definite are those which are equivalent to replacing certain of the $m$ variables by their negatives (that is, to reversing the directions of certain of the $m$ axes).

4. Superposability of finite subsets of $E_{n, r}$. A strictly elementary transformation of a matrix is one which is effected by (a) the interchange of two rows and the same numbered columns and/or (b) the multiplication of the elements of a row and those of the same numbered column by -1 . Two matrices are called strictly equivalent provided one may be transformed into the other by means of strictly elementary transformations.

Since $E_{n, r}$ is the metric transform of $S_{n, r}$ by the $\pi r$-supplementation function $F$, there corresponds to each ordered $m$-tuple $p_{1}, p_{2}, \cdots, p_{m}$ of $E_{n, r}$ a set of ordered $m$-tuples of $S_{n, r}$, each one of which is transformed into the ordered elliptic $m$-tuple by $F$. Each ordered $m$-tuple of the set is called an ordered associated spherical m-tuple of $p_{1}, p_{2}, \cdots, p_{m}$, and it is clear that if $p_{1}^{\prime}, p_{2}^{\prime}, \cdots, p_{m}^{\prime}$ is any one of these spherical $m$-tuples (that is, $\left(p_{1}, p_{2}, \cdots, p_{m}\right)=F\left(p_{1}^{\prime}, p_{2}^{\prime}, \cdots, p_{m}^{\prime}\right)$ with $\left.p_{i}^{\prime} \rightarrow p_{i}(i=1,2, \cdots, m)\right)$ then the set consists of all those $m$-tuples, free from diametral pointpairs, contained in the associated spherical $2 m$-tuple $p_{1}^{\prime \prime}, p_{2}^{\prime \prime}, \cdots, p_{m}^{\prime}, p_{1}^{*}, p_{2}^{*}, \cdots, p_{m}^{*}$ formed by adjoining those points $p_{i}^{*}$ of $S_{n, r}$ diametral to $p_{i}^{\prime}(i=1,2, \cdots, m)$.

Lemma 4.1. If $p_{1}^{\prime}, p_{2}^{\prime}, \cdots, p_{m}^{\prime}$ and $p_{1}^{\prime \prime}, p_{2}^{\prime \prime}, \cdots, p_{m}^{\prime \prime}$ are ordered associated spherical m-tuples of the elliptic subset $p_{1}, p_{2}, \cdots, p_{m}$ then the two matrices $\left(\cos p_{i}^{\prime} p_{j}^{\prime} / r\right)$ and $\left(\cos p_{i}^{\prime \prime} p_{j}^{\prime \prime} / r\right)(i, j=1,2, \cdots, m)$ are strictly equivalent.

Proof. It follows from the above that each point $p_{i}^{\prime \prime}$ of the second $m$-tuple is either identical with the corresponding point $p_{i}^{\prime}$ of the first $m$-tuple or is diametral to it. Hence the two sets may be labelled, respectively, $p_{i_{1}}^{\prime}, p_{i_{2}}^{\prime}, \cdots$, 
$p_{i_{k}}^{\prime}, p_{i_{k+1}}^{\prime}, \cdots, p_{i_{m}}^{\prime}$ and $p_{i_{1}}^{\prime \prime}, p_{k_{2}}^{\prime \prime}, \cdots, p_{i_{k}}^{\prime \prime}, p_{i_{k+1}}^{\prime \prime}, \cdots, p_{i_{m}}^{\prime \prime}$, with $p_{i_{1}}^{\prime \prime}=p_{i_{1}}^{\prime}$, $p_{k}^{\prime \prime}=p_{i_{2}}^{\prime}, \cdots, p_{i_{k}}^{\prime \prime}=p_{i_{k}}^{\prime}$ and $p_{i_{k+1}}^{\prime \prime}=p_{i_{k+1}}^{*}, \cdots, p_{t_{m}}^{\prime \prime}=p_{t_{m}}^{*} \quad(0 \leqq k \leqq m)$, where $p_{i j}^{*}$ is the point of $S_{n, r}$ diametral to $p_{i_{j}}^{\prime}(j=k+1, k+2, \cdots, m)$.

Forming the two symmetric matrices (cos $\left.p_{i_{0}}^{\prime} p_{t_{t}}^{\prime} / r\right),\left(\cos p_{t_{\mathrm{a}}}^{\prime \prime} p_{i_{t}}^{\prime \prime} / r\right)$ $(s, t=1,2, \cdots, m)$, we observe that, since $\cos \left(p_{t_{t}}^{\prime} p_{i_{t}}^{\prime} / r\right)=\cos \left(p_{t_{0}}^{\prime \prime} p_{t_{t}}^{\prime \prime} / r\right)$ for $(s, t=1,2, \cdots, k)$ as well as for $(s, t=k+1, k+2, \cdots, m)$, while the sum of the cosines is zero for $(s=1,2, \cdots, k ; t=k+1, k+2, \cdots, m)$ and $(s=k+1$, $k+2, \cdots, m ; t=1,2, \cdots, k)$, multiplication by -1 of the elements of the last $(m-k)$ rows and columns of one matrix transforms it into the other. Hence the matrices $\left(\cos p_{s_{a}}^{\prime} p_{t_{t}}^{\prime} / r\right),\left(\cos p_{t_{t}}^{\prime \prime} p_{t_{t}}^{\prime \prime} / r\right)(s, t=1,2, \cdots, m)$ are strictly equivalent and since these two matrices are strictly equivalent to $\left(\cos p_{i}^{\prime} p_{j}^{\prime} / r\right)$ and $\left(\cos p_{i}^{\prime \prime} p_{j}^{\prime \prime} / r\right)(i, j=1,2, \cdots, m)$, respectively, the lemma is proved.

The matrix $\left(\cos p_{i}^{\prime} p_{i}^{\prime} / r\right)(i, j=1,2, \cdots, m)$ of an ordered associated spherical $m$-tuple $p_{1}^{\prime}, p_{2}^{\prime}, \cdots, p_{m}^{\prime}$ of the ordered elliptic $m$-tuple $p_{1}, p_{2}, \cdots, p_{m}$ is called an associated matrix of the latter $m$-tuple. It is clear that $\left(\cos p_{i}^{\prime} p_{j}^{\prime} / r\right)=\left(\epsilon_{i j} \cos p_{i} p_{j} / r\right)(i, j=1,2, \cdots, m)$, where $\epsilon_{i j}=\epsilon_{j i}= \pm 1$, and we set $\epsilon_{i j}=1$ whenever $i=j$ or $p_{i} p_{j}=\pi r / 2(i, j=1,2, \cdots, m)$. To each $m$-tuple of $E_{n, r}$ is attached (1) the set of all spherical $m$-tuples which are associated with the orderings of the $m$-tuple, and (2) the set of all their associated matrices. Each matrix of this set is called an associated matrix of the $m$-tuple.

COROLlaRY. Each two associated matrices of an elliptic m-tuple are strictly equivalent.

The notion of associated matrix is used as the basis for superposability criteria, as instanced in the following theorem:

THEOREM 4.1. Two m-tuples of $E_{n, r}$ are superposable if and only if an associated matrix of one m-tuple is strictly equivalent to an associated matrix of the other.

Proof. To prove the necessity, let $\left(p_{1}, p_{2}, \cdots, p_{m}\right)$ and $\left(q_{1}, q_{2}, \cdots, q_{m}\right)$ be two superposable $m$-tuples of $E_{n, r}$ with the labelling selected so that

$$
p_{1}, p_{2}, \cdots, p_{m} \approx q_{1}, q_{2}, \cdots, q_{m} .
$$

If $\Gamma$ is a congruent mapping of $E_{n, r}$ onto itself, with $p_{i}=\Gamma\left(q_{i}\right)$ $(i=1,2, \cdots, m)$, then the associated spherical $2 m$-tuple $\left(q_{1}^{\prime}, q_{2}^{\prime}, \cdots\right.$, $\left.q_{m}^{\prime}, q_{1}^{*}, q_{2}^{*}, \cdots, q_{m}^{*}\right)$ is sent by $\Gamma$ into the associated spherical $2 m$-tuple $\left(p_{1}^{\prime}, p_{2}^{\prime}, \cdots, p_{m}^{\prime}, p_{1}^{*}, p_{2}^{*}, \cdots, p_{m}^{*}\right)$. Hence ordered associated spherical $m$-tuples of the two elliptic subsets exist with equal matrices, and the strict equivalence of associated matrices of the $m$-tuples follows from the preceding corollary.

Let, now, an associated matrix of $\left(p_{1}, p_{2}, \cdots, p_{m}\right)$ be strictly equivalent to 
an associated matrix of $\left(q_{1}, q_{2}, \cdots, q_{m}\right)$. Then an associated spherical $m$-tuple of the former subset is congruent with an associated spherical $m$-tuple of the latter, and hence a congruent mapping of $S_{n, r}$ onto itself exists which brings these two associated $m$-tuples into coincidence. This mapping induces a congruence of $E_{n, r}$ with itself which superposes the two elliptic $m$-tuples, and the theorem is established.

Remark 4.1. If $\left(p_{1}, p_{2}, p_{3}\right) \approx\left(q_{1}, q_{2}, q_{3}\right)$ and a pair of points of one triple has distance $\pi r / 2$, then the triples are superposable, for it is easily seen that associated matrices of the sets are strictly equivalent.

Remark 4.2. If $\left(p_{1}, p_{2}, p_{8}\right) \approx\left(q_{1}, q_{2}, q_{3}\right)$ and the determinant $\left|\epsilon_{i j} \cos p_{i} p_{i} / r\right|$ $(i, j=1,2,3), \epsilon_{i j}=-1(i \neq j)$, is negative, then the triples are superposable. For since $p_{1}, p_{2}, p_{3}$ and $q_{1}, q_{2}, q_{8}$ are elliptic subsets, the determinants of associated matrices are non-negative $\left.{ }^{8}\right)$ and hence $\left(\epsilon_{i j} \cos p_{i} p_{j} / r\right)(i, j=1,2,3)$, $\epsilon_{i j}=-1(i \neq j)$, is not an associated matrix of $p_{1}, p_{2}, p_{3}$, nor is $\left(\epsilon_{i j} \cos q_{i} q_{j} / r\right)$ $(i, j=1,2,3)$ an associated matrix of $q_{1}, q_{2}, q_{8}$, for the two triples are congruent. Since the determinants of strictly equivalent matrices are equal, no associated matrix of either of the triples is strictly equivalent to $\left(\epsilon_{i j} \cos p_{i} p_{j} / r\right)$ $(i, j=1,2,3), \epsilon_{i j}=-1 \quad(i \neq j)$. Then each associated matrix of each triple is strictly equivalent to the matrix $\left(\cos p_{i} p_{j} / r\right)(i, j=1,2,3)$, which proves the remark.

Remark 4.3. The result obtained in the preceding remark gives a precise meaning to such a statement as "congruent triples with diameter less than a fixed number, depending on the space, are superposable" sometimes encountered in the literature. Also, since the determinant $\left|\epsilon_{i j} \cos p_{i} p_{j} / r\right|$ $(i, j=1,2,3), \epsilon_{i j}=-1(i \neq j)$, is negative for a triple of $E_{n, r}$ if and only if $p_{1} p_{2}+p_{2} p_{3}+p_{1} p_{8}<\pi r$, a sufficient condition for the superposability of two congruent triples is simply that the sum of the three distances determined by the points of one of the triples be less than $\pi r$.

TheOREM 4.2. Let $p_{1}, p_{2}, \cdots, p_{m} \approx q_{1}, q_{2}, \cdots, q_{m}$ be two f-congruent $m$-tuples of $E_{n, r}$. The two ordered $m$-tuples are f-superposable if and only if there exists an associated matrix of one m-tuple which equals an associated matrix of the other.

Proof. If the two $m$-tuples are $f$-superposable, then ordered associated spherical $m$-tuples $p_{1}^{\prime}, p_{2}^{\prime}, \cdots, p_{m}^{\prime}$ and $q_{1}^{\prime}, q_{2}^{\prime}, \cdots, q_{m}^{\prime}$ exist with $p_{1}^{\prime}, p_{2}^{\prime}, \cdots, p_{m}^{\prime} \approx q_{1}^{\prime}, q_{2}^{\prime}, \cdots, q_{m}^{\prime}$. Then the two matrices (cos $\left.p_{i}^{\prime} p_{j}^{\prime} / r\right)$, $\left(\cos q_{i}^{\prime} q_{j}^{\prime} / r\right)(i, j=1,2, \cdots, m)$ are equal.

On the other hand, if an associated matrix of one of the ordered $m$-tuples equals an associated matrix of the other, then again associated spherical $m$-tuples exist with $p_{1}^{\prime}, p_{2}^{\prime}, \cdots, p_{m}^{\prime} \approx q_{1}^{\prime}, q_{2}^{\prime}, \cdots, q_{m}^{\prime}$. Since this is a congruence between two subsets of the spherical space $S_{n, r}$, it may be extended

(8) By the elliptic imbedding theorem [3, p. 336]. 
to the whole space; that is, a congruent mapping of $S_{n, r}$ onto itself exists which maps $p_{i}^{\prime}$ onto $q_{i}^{\prime}(i=1,2, \cdots, m)$. This mapping induces a congruence of $E_{n, r}$ with itself which maps $p_{i}$ onto $q_{i}(i=1,2, \cdots, m)$, and the two $m$-tuples are $f$-superposable.

THEOREM 4.3. If two triples of $E_{n, r}$ are superposable, any congruence between them can be extended to the whole space.

Proof. Let $\left(p_{1}, p_{2}, p_{3}\right) \cong\left(q_{1}, q_{2}, q_{3}\right)$ and suppose $p_{1}, p_{2}, p_{3} \approx q_{1}, q_{2}, q_{3}$. Selecting associated matrices $\left(\epsilon_{i j} \cos p_{i} p_{j} / r\right),\left(\epsilon_{i j}^{\prime} \cos q_{i} q_{j} / r\right)(i, j=1,2,3)$, with $\epsilon_{1 j}=\epsilon_{j 1}=\epsilon_{1 j}^{\prime}=\epsilon_{j 1}^{\prime}=1(j=1,2,3)$, it follows from the superposability of the two triples that these matrices are strictly equivalent. Then their determinants are equal, and expanding yields

$\epsilon_{23} \cos \left(p_{1} p_{2} / r\right) \cos \left(p_{2} p_{3} / r\right) \cos \left(p_{1} p_{3} / r\right)=\epsilon_{23}^{\prime} \cos \left(q_{1} q_{2} / r\right) \cos \left(q_{2} q_{3} / r\right) \cos \left(q_{1} q_{3} / r\right)$.

If neither $p_{1} p_{2}$ nor $p_{1} p_{3}$ equals $\pi r / 2$ then $\epsilon_{23}=\epsilon_{23}^{\prime} \quad\left(\epsilon_{23}=\epsilon_{23}^{\prime}=1\right.$ in case $\left.p_{2} p_{3}=\pi r / 2\right)$ and the two associated matrices are equal; while if $p_{1} p_{2}$ or $p_{1} p_{8}$ equals $\pi r / 2$ then the existence of equal associated matrices of the ordered triples $p_{1}, p_{2}, p_{3}$ and $q_{1}, q_{2}, q_{3}$ is obvious. Hence the two triples are $f$-superposable.

Remark 4.4. It is shown in $\$ 6$ that Theorem 4.3 cannot be extended to $m$-tuples, $m>3$; that is, superposability of two $m$-tuples $(m>3)$ of $E_{n, r}$ does not imply that each congruence between the $m$-tuples can be extended to the whole space.

TheOREM 4.4. Let $p_{1}, p_{2}, \cdots, p_{m} \approx f q_{1}, q_{2}, \cdots, q_{m}$ be two congruent subsets of $E_{n, r}$ such that (i) there exists a point $p_{k}$ of the first $m$-tuple with $p_{i} p_{k} \neq \pi r / 2$, $(i=1,2, \cdots, m)$ and (ii) each triple of $\left(p_{1}, p_{2}, \cdots, p_{m}\right)$ containing $p_{k}$ is superposable with the corresponding triple of $\left(q_{1}, q_{2}, \cdots, q_{m}\right)\left({ }^{9}\right)$. Then the two m-tuples are $f$-superposable.

Proof. Writing the above congruence in the form $p_{k}, p_{1}, p_{2}, \cdots, p_{k-1}$, $p_{k+1}, \cdots, p_{m} \approx q_{k}, q_{1}, q_{2}, \cdots, q_{k-1}, q_{k+1}, \cdots, q_{m}$ and making the cyclic substitution $(1,2, \cdots, k)$ on the first $k$ indices of both sets yields

$$
\begin{aligned}
p_{1}, p_{2}, \cdots, p_{m} & \approx f q_{1}, q_{2}, \cdots, q_{m}, \\
p_{1} p_{i} \neq \pi r / 2 & (i=1,2, \cdots, m) .
\end{aligned}
$$

Let $\left(\epsilon_{i j} \cos p_{i} p_{j} / r\right),\left(\epsilon_{i j}^{\prime} \cos q_{i} q_{j} / r\right)(i, j=1,2, \cdots, m)$ be two associated matrices of the respective ordered $m$-tuples, with $\epsilon_{1 j}=\epsilon_{i 1}=\epsilon_{1 j}^{\prime}=\epsilon_{j 1}^{\prime}=1$ $(j=2,3, \cdots, m)$. Since $\left(p_{1}, p_{s}, p_{t}\right) \cong\left(q_{1}, q_{s}, q_{t}\right)(s, t=2,3, \cdots, m)$, the respective third-order principal minors of the above matrices, which involve these triples, are strictly equivalent and hence the determinants of these minors are equal. Expanding and taking into account $\left({ }^{*}\right)$, together with

( $)$ Note that it is not assumed that the corresponding triples are $f$-superposable. 
$\epsilon_{i j}=1$ if $p_{i} p_{j}=\pi r / 2$, gives $\epsilon_{s t}=\epsilon_{s t}^{\prime}(s, t=2,3, \cdots, m)$. Then $\epsilon_{i j}=\epsilon_{i j}^{\prime}$ $(i, j=1,2, \cdots, m)$, and the two associated matrices are equal. Hence the ordered $m$-tuples are $f$-superposable.

Remark 4.5. The restriction imposed by hypothesis (i) of the theorem is essential, for if $p_{1} p_{s}=\pi r / 2$, the superposability of triples $p_{1}, p_{s}, p_{t}$ and $q_{1}, q_{s}, q_{t}$ does not imply that $\epsilon_{s t}=\epsilon_{s t}^{\prime}$.

Thus two congruent, non-superposable quadruples of $E_{2, r}$ exist with three pairs of corresponding triples superposable. Let, for example, $p_{4}, p_{4}^{\prime}$ be two distinct points of $E_{2, r}$ and $p_{1}, p_{2}, p_{3}$ a non- $E_{1, r}$ triple which is contained in the two mutually perpendicular lines of $E_{2, r}$ which form the locus of points equidistant from $p_{4}$ and $p_{4}^{\prime}$, with $p_{1}$ the pole of $E_{1, r}\left(p_{4}, p_{4}^{\prime}\right)$ and $p_{1} p_{3}, p_{1} p_{2}$ different from $\pi r / 2$. (In particular, the points may be selected so that $p_{1} p_{2}=p_{1} p_{3}=\pi r / 3, p_{2} p_{4}=p_{3} p_{4}=r \cdot \operatorname{arc} \cos 6^{1 / 2} / 4, p_{2} p_{3}=r \cdot \operatorname{arc} \cos 1 / 4, p_{1} p_{4}$ $=\pi r / 2$.) Then $p_{1}, p_{2}, p_{3}, p_{4} \approx p_{1}, p_{2}, p_{3}, p_{4}^{\prime}$ and the triple $p_{2}, p_{3}, p_{4}$ is the only triple of the first quadruple which is not superposable with the corresponding triple of the second.

It is proved later (Theorem 5.2) that any two subsets $P, Q$ of an elliptic space, with $P \sim f Q$, are $f$-superposable whenever each two corresponding finite subsets have that property. This result combined with the preceding theorem has as immediate consequence:

THEOREM 4.5. Let $P, Q$ be any two subsets of $E_{n, r}$ with $P \approx_{f} Q$ and such that no two points of $P$ have distance $\pi r / 2$. If each triple of $P$ is superposable with the corresponding triple of $Q$, then $P$ and $Q$ are $f$-superposable.

Thus if $P, Q \subset E_{n, r}$ with $P \sim_{f} Q$ and no two points of $P$ have distance $\pi r / 2$, then any congruence between these sets can be extended to a motion whenever $f$-corresponding triples of $P, Q$ are superposable.

In view of the hypotheses of Theorem 4.4 it is clear that the conclusion of the above theorem is valid under weaker assumptions than those that are made, but the cumbersome nature of the statement in terms of the weaker assumptions leads us to suppress its formulation.

THEOREM 4.6. Two f-congruent $m$-tuples

$$
p_{1}, p_{2}, \cdots, p_{m} \approx_{f} q_{1}, q_{2}, \cdots, q_{m}
$$

of $E_{n, r}$ are $f$-superposable if and only if, for a pair $p_{k}, q_{k}$ of corresponding points, arbitrarily small spherical neighborhoods $N\left(p_{k} ; \rho\right), N\left(q_{k} ; \rho\right)$ of radius $\rho$ exist with the property that corresponding to each point $p$ of $N\left(p_{k} ; \rho\right)$ with $p_{j} p \neq \pi r / 2$ $(j=1,2, \cdots, k-1, k+1, \cdots, m)$, there is a point $q$ of $N\left(q_{k} ; \rho\right)$ such that the two congruent m-tuples $p_{1}, p_{2}, \cdots, p_{k-1}, p, p_{k+1}, \cdots, p_{m} \approx q_{1}, q_{2}, \cdots, q_{k-1}$, $q, q_{k+1}, \cdots, q_{m}$ have those corresponding triples containing $p$ and $q$ superposable.

Proof. If $p_{1}, p_{2}, \cdots, p_{m} \cong_{f} q_{1}, q_{2}, \cdots, q_{m}$ then a motion $\Gamma$ of the space, with $\Gamma\left(p_{i}\right)=q_{i}(i=1,2, \cdots, m)$, carries arbitrarily small neighborhoods 
$N\left(p_{k} ; \rho\right)$ into congruent arbitrarily small neighborhoods $N\left(q_{k} ; \rho\right)$, and hence takes each $p$ of $N\left(p_{k} ; \rho\right)$ into a corresponding point $q$ of $N\left(q_{k} ; \rho\right)$. Then $p_{1}, p_{2}, \cdots, p_{k-1}, p, p_{k+1}, \cdots, p_{m} \cong q_{1}, q_{2}, \cdots, q_{k-1}, q, q_{k+1}, \cdots, q_{m}$ and the conclusion of the theorem is valid a fortiori.

Conversely, let $\left\{N_{i}\left(p_{k} ; \rho_{i}\right)\right\},\left\{N_{i}\left(q_{k} ; \rho_{i}\right)\right\}$ be sequences of neighborhoods of $p_{k}, q_{k}$, respectively, with $\rho_{i}<1 / i(i=1,2, \cdots)$, which have the properties stated in the theorem. Now each $N_{i}\left(p_{k} ; \rho_{i}\right)$ contains a point $p_{i}^{\prime}$ such that $p_{i}^{\prime} p_{j} \neq \pi r / 2(j=1,2, \cdots, k-1, k+1, \cdots, m)$, and hence a corresponding point $q_{i}^{\prime}$ of $N_{i}\left(q_{k} ; \rho_{i}\right)$ exists such that $p_{1}, p_{2}, \cdots, p_{k-1}, p_{i}^{\prime}, p_{k+1}, \cdots, p_{m}$ $\approx q_{1}, q_{2}, \cdots, q_{k-1}, q_{i}^{\prime}, q_{k+1}, \cdots, q_{m}$ with corresponding triples containing $p_{i}^{\prime}$ and $q_{i}^{\prime}$, respectively, superposable.

It follows from Theorem 4.4 that

$$
p_{1}, p_{2}, \cdots, p_{k-1}, p_{i}^{\prime}, p_{k+1}, \cdots, p_{m} \cong q_{1}, q_{2}, \cdots, q_{k-1}, q_{i}^{\prime}, q_{k+1}, \cdots, q_{m} ;
$$

and so an infinite sequence $\left\{\Gamma_{i}\right\}$ of motions exists with $\Gamma_{i}\left(p_{j}\right)=q_{i}$ $(j=1,2, \cdots, k-1, k+1, \cdots, m)$, and $\Gamma_{i}\left(p_{i}^{\prime}\right)=q_{i}^{\prime}(i=1,2, \cdots)$. From a theorem of van Dantzig and van der Waerden [4], the infinite sequence $\left\{\Gamma_{i}\right\}$ contains a convergent subsequence $\left\{\Gamma_{j_{i}}\right\}$ with a motion $\Gamma$ for limit; that is

$$
\begin{aligned}
\Gamma_{i_{i}}\left(p_{1}, p_{2}, \cdots, p_{k-1}, p_{i_{i}}^{\prime}, p_{k+1}, \cdots, p_{m}\right) & \\
& =\left(q_{1}, q_{2}, \cdots, q_{k-1}, q_{i_{i}}^{\prime}, q_{k+1}, \cdots, q_{m}\right)
\end{aligned}
$$

$(i=1,2, \cdots)$. Since $\lim _{i \rightarrow \infty} q_{j_{i}}^{\prime}=q_{k}, \lim _{i \rightarrow \infty} \Gamma_{j_{i}}\left(p_{j_{i}}^{\prime}\right)=\Gamma\left(p_{k}\right)$, then $\Gamma\left(p_{1}, p_{2}, \cdots\right.$, $\left.p_{k-1}, p_{k}, p_{k+1}, \cdots, p_{m}\right)=\left(q_{1}, q_{2}, \cdots, q_{k-1}, q_{k}, q_{k+1}, \cdots, q_{m}\right)$, and the theorem is proved $\left({ }^{10}\right)$.

5. Superposability of infinite subsets of $E_{n, r}$. Superposability order. In this section it is shown that the superposability of two congruent infinite subsets of $E_{n, r}$ is reducible to the superposability of corresponding finite subsets-in particular, to the superposability of corresponding $(k+1)$-tuples when the two sets lie in (coincident or distinct) $k$-dimensional subspaces. It will be seen that the number $k+1$ is the smallest possible.

THEOREM 5.1. Let $P, Q$ be two subsets of $E_{k, r}$ whose points are in a one-to-one correspondence such that each finite subset of $P$ is superposable with its corresponding subset of $Q$. Then this property can be extended to the closures $\bar{P}, \bar{Q}$ of the two subsets.

Proof. Since corresponding pairs of points of $P$ and $Q$ are superposable, the correspondence $P \sim Q$ between the two sets is a congruence $P \approx Q$, which can be extended in the usual manner to obtain $\bar{P} \approx \bar{Q}$.

Let $p_{1}, p_{2}, \cdots, p_{m}$ and $q_{1}, q_{2}, \cdots, q_{m}$ be corresponding $m$-tuples in the latter congruence. Then for each $p_{i}$ and $q_{i}$ infinite sequences $p_{i 1}, p_{i 2}, \cdots$ and

(10) Here $\Gamma\left(p_{i}\right)=q_{i}(i=1,2, \cdots, m)$. 
$q_{i 1}, q_{i 2}, \cdots$ of corresponding points of $P$ and $Q$, respectively, exist with $\lim _{n \rightarrow \infty} p_{i n}=p_{i}, \lim _{n \rightarrow \infty} q_{i n}=q_{i}(i=1,2, \therefore, m)$. If $\Gamma_{n}$ denotes the congruent mapping of $E_{k, r}$ onto itself which carries the points $p_{1 n}, p_{2 n}, \cdots, p_{m n}$ into the points $q_{1 n}, q_{2 n}, \cdots, q_{m n}$, respectively $(n=1,2, \cdots)$, it follows from the van Dantzig-van der Waerden theorem cited above that the infinite sequence $\left\{\Gamma_{n}\right\}$ of motions contains a subsequence $\left\{\Gamma_{j_{n}}\right\}$ with limit $\Gamma$, a motion.

If we select appropriate subsequences, $\lim _{n \rightarrow \infty} p_{i j_{n}}=p_{i}, \lim _{n \rightarrow \infty} q_{i j_{n}}=q_{i}$, and

$$
\begin{aligned}
\lim _{n \rightarrow \infty} \Gamma_{j_{n}}\left(p_{1 j_{n}}, p_{2 j_{n}}, \cdots, p_{m i_{n}}\right) & =\lim _{n \rightarrow \infty}\left(q_{1 i_{n}}, q_{2 i_{n}}, \cdots, q_{m j_{n}}\right) \\
& =\left(q_{1}, q_{2}, \cdots, q_{m}\right) .
\end{aligned}
$$

Now by the triangle inequality,

$$
\begin{aligned}
0 \leqq \Gamma_{j_{n}}\left(p_{i j_{n}}\right) \Gamma\left(p_{i}\right) & \leqq \Gamma_{j_{n}}\left(p_{i j_{n}}\right) \Gamma_{j_{n}}\left(p_{i}\right)+\Gamma_{j_{n}}\left(p_{i}\right) \Gamma\left(p_{i}\right) \\
& \leqq p_{i j_{n}} p_{i}+\Gamma_{j_{n}}\left(p_{i}\right) \Gamma\left(p_{i}\right)
\end{aligned}
$$

$(i=1,2, \cdots, m)$, and hence $\lim _{n \rightarrow \infty} \Gamma_{j_{i}}\left(p_{i j_{n}}\right) \Gamma\left(p_{i}\right)=0$, since $\lim _{n \rightarrow \infty} \Gamma_{i_{n}}(p)$ $=\Gamma(p)$ for every element $p$ of $E_{k, r}$.

Thus, $\lim _{n \rightarrow \infty} \Gamma_{j_{n}}\left(p_{i j_{n}}\right)=\Gamma\left(p_{i}\right) \quad(i=1,2, \cdots, m)$; that is, $\Gamma\left(p_{i}\right)=q_{i}$, $(i=1,2, \cdots, m)$, and the theorem is proved.

THEOREM 5.2. If $P, Q$ are two subsets of $E_{k, r}$ whose points are in a one-to-one correspondence $f$ such that each finite subset of $P$ is $f$-superposable with the corresponding subset of $Q$, then $P$ and $Q$ are $f$-superposable.

Proof. The theorem being trivial for $P$ and $Q$ finite subsets, suppose them infinite. As in the preceding theorem, the correspondence $P \sim f Q$ is seen to be a congruence $P \approx f Q$ which is extended to $\bar{P} \approx \bar{f} \bar{Q}$, where $\bar{f}$ is the extension of $f$ to $\bar{P}, \bar{Q}$.

Since $\bar{P}$ is a closed subset of the compact metric space $E_{k, r}$, it is the closure of one of its denumerable subsets, say $p_{1}, p_{2}, \cdots, p_{n}, \cdots$. Denoting by $q_{1}, q_{2}, \cdots, q_{n}, \cdots$ the corresponding subset of $\bar{Q}$, then for each positive integer $m$ a motion $\Gamma_{m}$ exists such that $\Gamma_{m}\left(p_{i}\right)=q_{i}(i=1,2, \cdots, m)$. Letting $\left\{\Gamma_{i_{m}}\right\} \rightarrow \Gamma$, it follows (since $\Gamma\left(p_{i}\right)=\lim _{m \rightarrow \infty} \Gamma_{i_{m}}\left(p_{i}\right)=q_{i}(i=1,2, \cdots)$ ) that $\bar{P} \cong_{\bar{f}} \bar{Q}$, for a motion which superposes the two denumerable subsets $p_{1}, p_{2}, \cdots, p_{n}, \cdots$ and $q_{1}, q_{2}, \cdots, q_{n}, \cdots$ evidently superposes their closures. This implies that $P$ and $Q$ are $f$-superposable.

THEOREM 5.3. If $P, Q$ are two finite subsets of $E_{k, r}$ with $P \approx f Q$ and such that each two corresponding $(k+2)$-tuples of $P$ and $Q$ are $f$-superposable, then $P$ and $Q$ are $f$-superposable.

Proof. If we suppose the sets to consist of $m+1$ points, $m>k+1$, it follows from the congruence of $E_{1, r}$ with $S_{1, r / 2}$, and the $f$-superposability of any two $f$-congruent subsets of $S_{1, r / 2}$, that the theorem is valid for $k=1$. An induc- 
tive hypothesis on the dimension $k$ establishes it whenever $P$ (and consequently $Q)$ lies in a $(k-1)$-dimensional hyperplane $E_{k-1, r}$, since a motion exists which will carry both sets into the same $E_{k-1, r}$.

Assume, then, that $P$ does not lie in any $E_{k-1, r}$, and make the inductive hypothesis of the theorem's validity for sets of $m$ points, the induction being anchored for $m=k+2$. Two cases are distinguished:

Case 1. There is a labelling $p_{1}, p_{2}, \cdots, p_{i}, p_{i+1}, \cdots, p_{m+1}$ of the points of $P$ such that there exists an integer $i(1 \leqq i \leqq m)$ for which each of the points $p_{i+1}, p_{i+2}, \cdots, p_{m+1}$ has distance $\pi r / 2$ from each of the points $p_{1}, p_{2}, \cdots, p_{i}$. Labelling the points of $Q$ so that $q_{j}=f\left(p_{j}\right)(j=1,2, \cdots, m+1)$, we have

$$
p_{1}, p_{2}, \cdots, p_{i}, p_{i+1}, \cdots, p_{m+1} \approx q_{1}, q_{2}, \cdots, q_{i}, q_{i+1}, \cdots, q_{m+1},
$$

and it is seen that any two associated matrices

$$
\left(\epsilon_{i j} \cos p_{i} p_{j} / r\right), \quad\left(\epsilon_{i j}^{\prime} \cos q_{i} q_{j} / r\right) \quad(i, j=1,2, \cdots, m+1)
$$

of $P, Q$, respectively, have all elements zero except those that are in the two blocks $(i \times i$ and $(m-i+1) \times(m-i+1))$ which occupy the upper left-hand and lower right-hand corners, respectively, of the two matrices. It follows at once from the inductive hypothesis and Theorem 4.2 that equal associated matrices of $P$ and $Q$ exist, and the two sets are $f$-superposable.

Case 2. The points of $P$ may not be labelled as in Case 1.

Then clearly a labelling $p_{i_{1}}, p_{i_{2}}, \cdots, p_{i_{m}}, p_{i_{m+1}}$ of the points of $P$ exists such that no point is a pole of each preceding point, and there is no difficulty in showing that these points may be rearranged so that this property is retained and in addition (since the $m+1$ points do not lie in an $E_{k-1, r}$ ) for each $i(1 \leqq i \leqq k+1)$ the first $i$ of the points determine a unique $E_{i-1, r}$ containing them.

Denoting such a rearrangement by $p_{1}, p_{2}, \cdots, p_{k}, p_{k+1}, \cdots, p_{m+1}$, and labelling the points of $Q$ so that $q_{i}=f\left(p_{i}\right)(i=1,2, \cdots, m+1)$, we have

$$
p_{1}, p_{2}, \cdots, p_{k}, p_{k+1}, \cdots, p_{m+1} \approx f, q_{1}, q_{2}, \cdots, q_{k}, q_{k+1}, \cdots, q_{m+1},
$$

with both $(m+1)$-tuples in apolar order.

Let, now, $\Gamma$ be a motion such that $\Gamma\left(q_{i}\right)=p_{i}(i=1,2, \cdots, m-1, m+1)$, and let $\Gamma\left(q_{m}\right)=\bar{p}_{m}$. Then

$$
p_{1}, p_{2}, \cdots, p_{m-1}, p_{m} \cong_{f} q_{1}, q_{2}, \cdots, q_{m-1}, q_{m} \cong_{f} p_{1}, p_{2}, \cdots, p_{m-1}, p_{m} .
$$

But since $m-1 \geqq k+1$, and $p_{1}, p_{2}, \cdots, p_{k+1}$ do not lie in any $E_{k-1, r}$, it follows from the apolar ordering that the identity is the only motion which superposes the first and third $m$-tuples. Hence $p_{m}=p_{m}$ and $P \cong_{f} Q$.

Before proving the final reduction theorem, it is convenient to establish two lemmas.

Lemma, 5.1. Let $P$ be a set of $k+2$ points of $E_{k, r}$, with no $(k+1)$-tuple in a 
hyperplane $E_{k-1, r}$, and let $Q$ be a subset of $E_{k, r}$ whose points are in a one-to-one correspondence $f$ with those of $P$. If (i) each two corresponding $(k+1)$-tuples of $P$ and $Q$ are $f$-superposable and (ii) $P$ and $Q$ are not $f$-superposable, then in each right-hand apolar ordering of $P$, either the first point or the second point has distance $\pi r / 2$ from each of the last $k-1$ points.

Proof. Let $p_{1}, p_{2}, \cdots, p_{k}, p_{k+1}, p_{k+2}$ be any right-hand apolar ordering of $P$ and suppose $\Gamma\left(q_{i}\right)=p_{i}(i=2,3, \cdots, k+2)$, while $\Gamma\left(q_{1}\right)=p_{1} \neq p_{1}$.

Since $p_{1}, p_{3}, \cdots, p_{k}, p_{k+1}, p_{k+2} \cong q_{1}, q_{3}, \cdots, q_{k}, q_{k+1}, q_{k+2} \cong p_{1}, p_{3}, \cdots$, $p_{k}, p_{k+1}, p_{k+2}$, it follows from the apolar ordering of $P$ that the hyperplane $E_{k-1, r}\left(p_{3}, p_{4}, \cdots, p_{k+1}, p_{k+2}\right)$ remains pointwise fixed under a motion which superposes the first and third of the above $(k+1)$-tuples. Hence this hyperplane is part of the locus of points $E_{k, r}$ equidistant from $p_{1}$ and $p_{1}$.

In similar manner

$$
p_{1}, p_{2}, p_{4}, \cdots, p_{k+1}, p_{k+2} \cong p_{1}, p_{2}, p_{4}, \cdots, p_{k+1}, p_{k+2},
$$

and if $p_{2}$ does not have distance $\pi r / 2$ from each of the points $p_{4}, p_{5}, \cdots, p_{k+2}$ then $E_{k-1, r}\left(p_{2}, p_{4}, \cdots, p_{k+1}, p_{k+2}\right)$ also forms part of the same equidistant locus. Since these two hyperplanes are distinct (otherwise $p_{2}, p_{3}, \cdots$, $p_{k+1}, p_{k+2}$ lie in an $\left.E_{k-1, r}\right)$ they intersect in the $E_{k-2, r}\left(p_{4}, p_{5}, \cdots, p_{k+2}\right)$ which is the absolute polar subspace of the line $E_{1, r}\left(p_{1}, p_{1}\right)$. Hence $p_{1} p_{4}=p_{1} p_{6}$ $=\cdots=p_{1} p_{k+2}=\pi r / 2$, and the lemma is established.

Remark 5.1. Though the hypothesis of Lemma 5.1 assumes that each two corresponding $(k+1)$-tuples of $P$ and $Q$ are $f$-superposable, the proof makes use of only three pairs of $f$-superposable $(k+1)$-tuples. Hence a somewhat stronger property than that asserted is proved.

Lemma 5.2. Let $P$ be a set of $k+2$ points of $E_{k, r}$, with no $(k+1)$-tuple in a hyperplane $E_{k-1, r} . A$ right-hand apolar ordering of the points of $P$ exists such that the distance between the third and the last points is not $\pi r / 2$.

Proof. To show that at least one right-hand apolar ordering of $P$ exists, let $p_{i+1}, p_{i+2}, \cdots, p_{k+1}, p_{k+2}$ be $k+2-i$ points of $P$ in right-hand apolar order. Then at least one of the remaining points of $P$ exists (label it $p_{i}$ ) such that $p_{i}, p_{i+1}, p_{i+2}, \cdots, p_{k+1}, p_{k+2}$ are in right-hand apolar order, for in the contrary case all of the remaining $i$ points of $P$ lie in the hyperplane $E_{i-2, r}$ of $(i-2)$ dimensions, which is the absolute polar of $E_{k-i+1, r}\left(p_{i+1}, p_{i+2}, \cdots, p_{k+2}\right)$. But then $k+1$ points of $P$ lie in an $E_{k-1, r}$, contrary to the hypothesis.

Since $P$ is a $(k+2)$-tuple of $E_{k, r}$, at least one pair of its points, say $p_{k+1}, p_{k+2}$, has distance different from $\pi r / 2$, and so $p_{k+1}, p_{k+2}$ are in right-hand apolar order. It follows, then, that a right-hand apolar ordering of $P$ exists.

Let, now, $p_{1}, p_{2}, p_{3}, p_{4}, \cdots, p_{k+1}, p_{k+2}$ be a right-hand apolar ordering of $P$. Then at least one of the points $p_{4}, p_{5}, \cdots, p_{k+2}$ has distance from $p_{3}$ different from $\pi r / 2$. Let $p_{i_{1}}$ be the point with greatest index $\left(3<i_{1} \leqq k+2\right)$ 
such that $p_{3} p_{i_{1}} \neq \pi r / 2$. If $i_{1}<k+2$, denote by $p_{i_{2}}\left(i_{1}<i_{2} \leqq k+2\right)$ the point of greatest index such that $p_{i_{1}} p_{i_{2}} \neq \pi r / 2$.

After $m$ applications of this process, the point $p_{k+2}$ is reached as the point of greatest index such that $p_{i_{m}} p_{k+2} \neq \pi r / 2$. Then the ordering $p_{1}, p_{2}, p_{3}, \cdots$, $p_{i_{1}-1}, p_{i_{1}+1}, \cdots, p_{i_{2}-1}, p_{i_{2}+1}, \cdots, p_{i_{m}-1}, p_{i_{m}+1}, \cdots, p_{k+2}, p_{i_{m}}, p_{i_{m-1}}, \cdots$, $p_{i_{2}}, p_{i_{1}}$ is seen at once to be a right-hand apolar ordering with $p_{3} p_{i_{1}} \neq \pi r / 2$, and the lemma is proved.

TheOREM 5.4. If $P, Q$ are two $(k+2)$-tuples of $E_{k, r}$ whose points are in a one-to-one correspondence $f$ such that each set of $k+1$ points of $P$ is $f$-superposable with the corresponding set of $k+1$ points of $Q$, then $P$ and $Q$ are $f$-superposable.

Proof. Make the inductive hypothesis, anchored for $k=1$, of the theorem's validity in each proper subspace of $E_{k, r}$ and distinguish two cases:

Case 1. $P$ contains a set of $k+1$ points which lies in a hyperplane $E_{k-1, r}$.

If the labelling be selected so that $p_{1}, p_{2}, \cdots, p_{k+1}$ is such a subset of $P$, then the inductive hypothesis establishes the theorem unless $p_{1}, p_{2}, \cdots, p_{k+1}$ determine a (unique) $E_{k-1, r}$, and this hyperplane does not contain the remaining point $p_{0}$.

Writing $q_{i}=f\left(p_{i}\right)(i=0,1, \cdots, k+1)$, let $p$ and $q$ denote the feet of $p_{0}$ and $q_{0}$, respectively, on the hyperplanes $E_{k-1, r}\left(p_{1}, p_{2}, \cdots, p_{k+1}\right), E_{k-1, r}\left(q_{1}\right.$, $\left.q_{2}, \cdots, q_{k+1}\right)$. If $p$ and $q$ are not unique, then $p_{0}$ and $q_{0}$ are poles of the (respective) hyperplanes, and a motion which superposes $p_{1}, p_{2}, \cdots, p_{k+1}$ and $q_{1}, q_{2}, \cdots, q_{k+1}$ evidently makes $p_{0}$ and $q_{0}$ coincide. In the event $p_{0}$ and $q_{0}$ are unique, we prove the following assertion.

Assertion. $p, p_{1}, p_{2}, \cdots, p_{k+1} \cong q, q_{1}, q_{2}, \cdots, q_{k+1}$.

Since these two $(k+2)$-tuples lie in $(k-1)$-dimensional subspaces their superposability follows from the inductive hypothesis and the preceding theorem provided each two corresponding $k$-tuples are superposable (in the order indicated). Since $p_{1}, p_{2}, \cdots, p_{k+1} \cong q_{1}, q_{2}, \cdots, q_{k+1}$, it suffices to consider only those corresponding $k$-tuples that contain $p$ and $q$. Let $p, p_{i_{1}}$, $p_{i_{2}}, \cdots, p_{i_{k-1}}$ be such a $k$-tuple. If $p_{i_{1}}, p_{i_{2}}, \cdots, p_{i_{k-1}}, p_{i_{k}}$ are not in an $E_{k-2, r}$, a motion $\Gamma$ such that $\Gamma\left(p_{0}\right)=q_{0}, \Gamma\left(p_{i j}\right)=q_{i j}(j=1,2, \cdots, k)$ carries $E_{k-1, r}\left(p_{1}, p_{2}, \cdots, p_{k+1}\right)$ into $E_{k-1, r}\left(q_{1}, q_{2}, \cdots, q_{k+1}\right)$ and hence $\Gamma(p)=q$, and the assertion is proved.

If, on the other hand, $p_{i_{1}}, p_{i_{2}}, \cdots, p_{i_{k-1}}, p_{i_{k}} \in E_{k-2, r}$ while $p_{i_{1}}, p_{i_{2}}, \cdots$, $p_{i_{k-1}}, p_{i_{k+1}}$ are not points of any $E_{k-2, r}$, a repetition of the above argument for this latter set establishes the assertion. If, finally, $p_{i_{1}}, p_{i_{2}}, \cdots, p_{i_{k-1}}, p_{i j}$ belong to $(k-2)$-dimensional subspaces for $j=k$ and $j=k+1$, then $p_{i_{1}}, p_{i_{2}}, \cdots, p_{i_{k-2}}, p_{i_{k-1}} \in E_{k-3, r}$, since otherwise the $(k+2)$-tuple $p_{0}, p_{1}, \cdots$, $p_{k+1}$ would lie in an $E_{k-1, r}$.

To show $p, p_{i_{1}}, p_{i_{2}}, \cdots, p_{i_{k-1}} \cong q, q_{i_{1}}, q_{i_{2}}, \cdots, q_{i_{k-1}}$ in this event, it suffices to show that each $(k-1)$-tuple of the first set (which contains $p$ ) is 
superposable with the corresponding $(k-1)$-tuple of the second set.

Consider, for example, the $(k-1)$-tuple $p, p_{i_{1}}, p_{i_{2}}, \cdots, p_{i_{k-2}}$. The points $p_{i_{1}}, p_{i_{2}}, \cdots, p_{i_{k-2}}, p_{i_{k}}, p_{i_{k+1}}$ do not lie in any $E_{k-2, r}$, for if they did then $p_{i_{k-1}}$ would belong to that $E_{k-2, r}$ (since it belongs to a $(k-3)$-dimensional subspace of it) and hence the $k+2$ points are in an $E_{k-1, r}$. It follows that a motion $\Gamma$ such that

$$
\Gamma\left(p_{0}, p_{i_{1}}, \cdots, p_{i_{k-2}}, p_{i_{k}}, p_{i_{k+1}}\right)=\left(q_{0}, q_{i_{1}}, \cdots, q_{i_{k-2}}, q_{i_{k}}, q_{i_{k+1}}\right)
$$

(with corresponding points in the order indicated) carries $E_{k-1, r}\left(p_{1}\right.$, $\left.p_{2}, \cdots, p_{k+1}\right)$ into $E_{k-1, r}\left(q_{1}, q_{2}, \cdots, q_{k+1}\right)$ and hence $\Gamma(p)=q$.

In a similar manner, the other pairs of corresponding $(k-1)$-tuples are seen to be superposable, and so $p, p_{i_{1}}, p_{i_{2}}, \cdots, p_{i_{k-1}} \cong q, q_{i_{1}}, q_{i_{2}}, \cdots, q_{i_{k-1}}$, from which the assertion follows.

Let, now, $\Gamma$ be any motion such that $\Gamma\left(p_{i}\right)=q_{i}(i=1,2, \cdots, k+1)$, $\Gamma(p)=q$. Since $p_{1}, p_{2}, \cdots, p_{k+1} \in E_{k-1, r}$ (and not points of $E_{k-2, r}$ ) then $\Gamma\left(p_{0}\right)=q_{0}$ or $\Gamma\left(p_{0}\right)=\bar{q}_{0}$, the reflection of $q_{0}$ in $E_{k-1, r}\left(q_{1}, q_{2}, \cdots, q_{k+1}\right)$. In the latter event, rotation of $E_{k, r} 180$ degrees about the absolute pole of $E_{k-1, r}\left(q_{1}\right.$, $\left.q_{2}, \cdots, q_{k+1}\right)$ accomplishes the desired result.

Case 2. No $(k+1)$-tuple of $P$ lies in a hyperplane $E_{k-1, r}$.

Then each $i+1$ of the points of $P(1 \leqq i \leqq k)$ determines a unique $i$-dimensional subspace $E_{i, r}$ containing them.

Assuming that the two $(k+2)$-tuples $P, Q$ are not superposable $f$, let the labelling of the points of $P$ be chosen so that

$$
p_{1}, p_{2}, p_{k+2}, p_{3}, \cdots, p_{k}, p_{k+1}
$$

is a right-hand apolar ordering of $P$ with $p_{k+1} p_{k+2} \neq \pi r / 2$ (Lemma 5.2). Then by Lemma 5.1 either

$$
p_{1} p_{3}=p_{1} p_{4}=\cdots=p_{1} p_{k}=p_{1} p_{k+1}=\pi r / 2,
$$

or $p_{2} p_{3}=p_{2} p_{4}=\cdots=p_{2} p_{k}=p_{2} p_{k+1}=\pi r / 2$.

It is clear that if the first set of relations do not hold, another apolar ordering of the desired kind is obtained from $\left(^{*}\right)$ by interchanging the points $p_{1}, p_{2}$. Then interchanging their labellings restores the apolar ordering $\left({ }^{*}\right)$. We may, therefore, suppose relations $\left({ }^{* *}\right)$ and ordering $(*)$.

If we label the points of $Q$ so that $q_{i}=f\left(p_{i}\right)(i=1,2, \cdots, k+2)$, it follows easily from a comparison of two associated matrices of $P$ and $Q$, and the assumption that the two $(k+2)$-tuples are not $f$-superposable, that

$$
p_{1} p_{2} \neq \pi r / 2 \neq p_{1} p_{k+2}\left({ }^{11}\right) \text {. }
$$

(11) Also, if, for example, $p_{1} p_{2}=\pi r / 2$ then $p_{1}$ is the absolute pole of the $E_{k-1, r}$ determined by the points $p_{2}, p_{2}, \cdots, p_{k+1}$ and a motion which superposes the two $(k+1)$-tuples $p_{2}$, $p_{3}, \cdots, p_{k+1}, p_{k+2}$ and $q_{2}, q_{8}, \cdots, q_{k+1}, q_{k+2}$ clearly brings $p_{1}$ and $q_{1}$ into coincidence. 
Since $p_{k+1} p_{k+2} \neq \pi r / 2$, the arrangement $p_{1}, p_{2}, p_{3}, \cdots, p_{k+1}, p_{k+2}$ is a right-hand apolar ordering, and if we use Lemma 5.1 (together with $\left.p_{1} p_{k+2} \neq \pi r / 2\right)$ it follows that $p_{2} p_{4}=p_{2} p_{5}=\cdots=p_{2} p_{k+2}=\pi r / 2$, while, as before, $p_{2} p_{3} \neq \pi r / 2$. Similarly, from the apolar ordering $p_{2}, p_{3}, p_{4}, \cdots, p_{k+1}$, $p_{k+2}, p_{1}$ follows $p_{3} p_{5}=p_{3} p_{6}=\cdots=p_{3} p_{k+2}=p_{3} p_{1}=\pi r / 2$, and $p_{3} p_{4} \neq \pi r / 2$.

If we assume $p_{1} p_{2}, p_{2} p_{3}, \cdots, p_{i-1} p_{i}$ each different from $\pi r / 2$, and each of the points $p_{1}, p_{2}, \cdots, p_{i-1}$ with distance $\pi r / 2$ from all points of $P$ except their immediate predecessor and successor $\left(p_{0}=p_{k+2}\right)$, then

$$
p_{i-1}, p_{i}, p_{i+1}, p_{i+2}, \cdots, p_{k+2}, p_{1}, p_{2}, \cdots, p_{i-2}
$$

are in right-hand apolar order, and since $p_{i-1} p_{i-2} \neq \pi r / 2$, Lemma 5.1 yields

$$
p_{i} p_{i+2}=p_{i} p_{i+3}=\cdots=p_{i} p_{k+2}=p_{i} p_{1}=p_{i} p_{2}=\cdots=p_{i} p_{i-2}=\pi r / 2,
$$

while, since $P$ and $Q$ are assumed not $f$-superposable, $p_{i} p_{i+1} \neq \pi r / 2$.

Thus each point $p_{i}$ of $P(1 \leqq i \leqq k+2)$ has distance $\pi r / 2$ from exactly $k-1$ points of $P$, and $p_{i-1} p_{i} \neq \pi r / 2 \neq p_{i} p_{i+1}\left(p_{0}=p_{k+2}, p_{1}=p_{k+3}\right)$ with the corresponding statement valid for the set $Q$, since $P$ and $Q$ are $f$-congruent.

Consider, now, two associated matrices $\left(\epsilon_{i j} \cos p_{i} p_{j} / r\right),\left(\epsilon_{i j}^{\prime} \cos q_{i} q_{j} / r\right)$ $(i, j=1,2, \cdots, k+2)$ of the ordered $(k+2)$-tuples $P$ and $Q$, respectively. From the distance relations obtained above, the $f$-superposability of corresponding $(k+1)$-tuples, and the non- $f$-superposability of $P$ and $Q$, it follows that these two matrices may be selected so that $\epsilon_{i j}=\epsilon_{i j}^{\prime}=1$ $(i, j=1,2, \cdots, k+2)$, except that $\epsilon_{1, k+2}^{\prime}=-1=\epsilon_{k+2,1}^{\prime}$.

Since $P$ and $Q$ are $(k+2)$-tuples of $E_{k, r}$, the determinants of these matrices vanish. Expanding each determinant as a quadratic function of the elements in the last row (method of Cauchy) and equating, we see after easy computation that the product

$$
\cos \left(p_{1} p_{2} / r\right) \cdot \cos \left(p_{2} p_{3} / r\right) \cdots \cdots \cos \left(p_{k+1} p_{k+2} / r\right) \cdot \cos \left(p_{1} p_{k+2} / r\right)
$$

vanishes.

Hence one of the distances $p_{i} p_{i+1}(i=1,2, \cdots, k+2)$ equals $\pi r / 2$, contrary to what has been shown. This contradiction implies that $P$ and $Q$ are $f$-superposable, and the proof of the theorem is complete.

Combining the last three theorems yields the final reduction theorem.

THEOREM 5.5. If $P, Q$ are any two subsets of a $k$-dimensional elliptic space $E_{k, r}$, whose points are in a one-to-one correspondence $f$ such that each two corresponding $(k+1)$-tuples are $f$-superposable, then $P$ and $Q$ are $f$-superposable.

It is observed that since any two elliptic subspaces of the same dimension are superposable, the above theorem is valid whether or not $P$ and $Q$ lie in the same $E_{k, r}$.

Definition. An elliptic space has superposability order $\sigma$ provided any two subsets of the space are superposable whenever a one-to-one cor- 
respondence $f$ exists between the points of the two sets such that each two corresponding $\sigma$-tuples are $f$-superposable.

THEOREM 5.6. The k-dimensional elliptic space $E_{k, r}$ has the minimum superposability order $k+1$.

Proof. That $E_{k, r}$ has superposability order $k+1$ follows at once from Theorem 5.5. To show this a minimum it suffices to prove the existence in $E_{k, r}$ of two non-superposable $(k+1)$-tuples $p_{1}, p_{2}, \cdots, p_{k+1}$ and $q_{1}, q_{2}, \cdots$, $q_{k+1}$ such that $p_{1}, p_{2}, \cdots, p_{i-1}, p_{i+1}, \cdots, p_{k+1} \cong q_{1}, q_{2}, \cdots, q_{i-1}, q_{i+1}, \cdots$, $q_{k+1}(i=1, \dot{2}, \cdots, k+1)$.

Consider the two determinants $\Delta_{1}=\left|d_{i j}\right|, d_{i j}=d_{j i}=d,|i-j|=1$ or $k$, $d_{i i}=1$, and $d_{i j}=0$ for all remaining elements $(i, j=1,2, \cdots, k+1)$, and $\Delta_{2}=\left|d_{i j}^{\prime}\right|$, where the $d_{\imath \jmath}^{\prime}$ satisfy all the above conditions except that $d_{1, k+1}^{\prime}$ $=d_{k+1,1}^{\prime}=-d$. Since each principal minor in both of these determinants equals 1 for $d=0$, it follows from continuity considerations that a number $d$ exists $(0<d<1)$ such that all of the principal minors of both determinants are positive.

This implies that the $k$-dimensional spherical space $S_{k, r}$ contains two $(k+1)$-tuples $p_{1}^{\prime}, p_{2}^{\prime}, \cdots, p_{k+1}^{\prime}$ and $q_{1}^{\prime}, q_{2}^{\prime}, \cdots, q_{k+1}^{\prime}$ with $p_{i}^{\prime} p_{j}^{\prime}=r \cdot \arccos (d)$ $=q_{i}^{\prime} q_{j}^{\prime}, \quad|i-j|=1, \quad p_{i}^{\prime} p_{j}^{\prime}=\pi r / 2=q_{i}^{\prime} q_{j}^{\prime},|i-j| \neq 1 \quad(i, j=1,2, \cdots, k+1$; $i, j \neq 1, k+1), p_{1}^{\prime} p_{k+1}^{\prime}=r \cdot \operatorname{arc} \cos (d), q_{1}^{\prime} q_{k+1}^{\prime}=\pi r-r \cdot \operatorname{arc} \cos (d)$.

Passing to the $k$-dimensional elliptic space $E_{k, r}$, we obtain two congruent $(k+1)$-tuples $p_{1}, p_{2}, \cdots, p_{k+1}$ and $q_{1}, q_{2}, \cdots, q_{k+1}$ with associated matrices (the matrices of $\Delta_{1}$ and $\Delta_{2}$, respectively) which are clearly not strictly equivalent, though for each two corresponding $k$-tuples equal associated matrices (corresponding $k$ th order principal minors) may be selected. Hence each two corresponding $k$-tuples of the two $(k+1)$-tuples are $f$-superposable (where $\left.q_{i}=f\left(p_{i}\right)(i=1,2, \cdots, k+1)\right)$ while the two $(k+1)$-tuples are not superposable. This establishes the theorem.

6. Pseudo $f$-superposable sets. Concluding remarks. In the proof of Theorem 5.6 there was shown to exist two $(k+1)$-tuples of $E_{k, r}$ which are not $f$-superposable though each two corresponding $k$-tuples are. This circumstance gives rise to the following definition.

Definition. Two subsets of $E_{k, r}$ are called pseudo f-superposable of degree $k$ provided a one-to-one correspondence $f$ exists between their points such that (1) each two corresponding $k$-tuples are $f$-superposable and (2) the two subsets are not $f$-superposable. exist.

THEOREM 6.1. For each integer $k$, pseudo f-superposable sets of degree $k$

Proof. Such sets are instanced in the preceding theorem.

Remark 6.1. If $p_{1}, p_{2}, \cdots, p_{k+1}$ and $q_{1}, q_{2}, \cdots, q_{k+1}$ are pseudo $f$-superposable of degree $k$ then no $k$-tuple of either set lies in an $E_{k-2, r}$, for if either set contained such a $k$-tuple then that $k$-tuple of the other set superposable 
with it would also lie in an $E_{k-2, r}$, and consequently both $(k+1)$-tuples would be subsets of $(k-1)$-dimensional subspaces. Superposing these two subspaces yields two $(k+1)$-tuples of an $E_{k-1, r}$ with corresponding $k$-tuples $f$-superposable, and hence (Theorem 5.4) the $(k+1)$-tuples are $f$-superposable; that is $p_{1}, p_{2}, \cdots, p_{k+1}$ and $q_{1}, q_{2}, \cdots, q_{k+1}$ are $f$-superposable, contrary to assumption.

Remark 6.2. Consider four points $p_{1}^{\prime}, p_{2}^{\prime}, p_{3}^{\prime}, p_{4}^{\prime}$ of $S_{2, r}$ with $p_{1}^{\prime} p_{2}^{\prime}=p_{2}^{\prime} p_{3}^{\prime}$ $=p_{3}^{\prime} p_{4}^{\prime}=p_{1}^{\prime} p_{4}^{\prime}=\pi r / 3, p_{1}^{\prime} p_{3}^{\prime}=p_{2}^{\prime} p_{4}^{\prime}=\pi r / 2$ and four points $q_{1}^{\prime}, q_{2}^{\prime}, q_{3}^{\prime}, q_{4}^{\prime}$ of $S_{3, r}$ (not of $S_{2, r}$ ) with $q_{i}^{\prime} q_{j}^{\prime}=p_{i}^{\prime} p_{j}^{\prime}$, except that $q_{1}^{\prime} q_{4}^{\prime}=2 \pi r / 3\left({ }^{12}\right)$. Passing to the elliptic space $E_{3, r}$ yields two quadruples $p_{1}, p_{2}, p_{3}, p_{4}$ and $q_{1}, q_{2}, q_{3}, q_{4}$ which are pseudo $f$-superposable of degree 3 , with the first quadruple in $E_{2, r}$ and the second in $E_{3, r}$ (not in $E_{2, r}$ ). Hence of two pseudo $f$-superposable $(k+1)$-tuples of degree $k$, one might lie in an $E_{k-1, r}$.

Using Remark 6.1 and the method of Case 2, Theorem 5.4, the following theorem may be obtained.

Theorem 6.2 (Characterization of PSEUdo $f$-SUPerposable $(k+1)$ TUPLES OF DEGREE $k>1$ ). Two elliptic $(k+1)$-tuples $p_{1}, p_{2}, \cdots, p_{k+1}$ and $q_{1}, q_{2}, \cdots, q_{k+1}$ are pseudo $f$-superposable of degree $k>1$ if and only if associated matrices $\left(\phi_{i j}\right)=\left(\epsilon_{i j} \cos p_{i} p_{j} / r\right),\left(\phi_{i j}^{\prime}\right)=\left(\epsilon_{i j}^{\prime} \cos q_{i} q_{j} / r\right)(i, j=1,2, \cdots$, $k+1)$ of the respective ordered $(k+1)$-tuples exist such that $\phi_{i j}=\phi_{i j}^{\prime}>0,|i-j|=1$, $\phi_{i j}=\phi_{i j}^{\prime}=0,|i-j| \neq 1(i \neq j ; i, j=1,2, \cdots, k+1 ; i, j \neq 1, k+1), 0<\phi_{1, k+1}$ $=-\phi_{1, k+1}^{\prime}$.

Thus none of the sides $p_{i} p_{i+1}$ of the ordered $(k+1)$-gon $p_{1}, p_{2}, \cdots, p_{k+1}$ equals $\pi r / 2$ while each of the diagonals does. The ordered $(k+1)$-gon $q_{1}, q_{2}, \cdots, q_{k+1}$ congruent to $p_{1}, p_{2}, \cdots, p_{k+1}$ has spherical distance $\left(q_{i}, q_{j}\right)$ equal to spherical distance $\left(p_{i}, p_{j}\right)$ except that the spherical distances of $q_{1}, q_{k+1}$ and $p_{1}, p_{k+1}$ are supplementary.

We have seen that while euclidean, spherical, and hyperbolic spaces have the property that any congruence between two subsets can be extended to the whole space, subsets of elliptic space exist such that no congruence between them can be extended to the whole space ( $\$ 3)$.

It might be asked, however, whether a vestige of the property of free superposability is present in elliptic space in that subsets which are superposable are freely superposable. That is, if there exists a congruence between two elliptic subsets which can be extended to the whole space, does every congruence between the sets have this property?

That this question must be answered in the negative is shown by the interesting example of two quintuples in $E_{3, r}$ which are $g$-superposable but not $f$-superposable-indeed, the two quintuples are pseudo $f$-superposable. It is easy to prove that the $S_{3, r}$ contains two quintuples $p_{1}^{\prime}, p_{2}^{\prime}, \cdots, p_{5}^{\prime}$ and

(12) The two quadruples are easily shown to exist. 
$q_{1}^{\prime}, q_{2}^{\prime}, \cdots, q_{5}^{\prime}$ with

$$
\begin{aligned}
& \cos \left(p_{1}^{\prime} p_{2}^{\prime} / r\right)=\cos \left(p_{1}^{\prime} p_{4}^{\prime} / r\right)=\cos \left(p_{2}^{\prime} p_{3}^{\prime} / r\right)=a, \quad 0<a<1, \\
& \cos \left(p_{1}^{\prime} p_{3}^{\prime} / r\right)=\cos \left(p_{1}^{\prime} p_{5}^{\prime} / r\right)=\cos \left(p_{2}^{\prime} p_{4}^{\prime} / r\right)=\cos \left(p_{3}^{\prime} p_{5}^{\prime} / r\right)=0, \\
& \cos \left(p_{2}^{\prime} p_{5}^{\prime} / r\right)=\cos \left(p_{3}^{\prime} p_{4}^{\prime} / r\right)=b, \quad 0<b<1, \quad \cos \left(p_{4}^{\prime} p_{5}^{\prime} / r\right)=-a,
\end{aligned}
$$

the mutual distances of $q_{1}^{\prime}, q_{2}^{\prime}, \cdots, q_{5}^{\prime}$ such that $q_{i}^{\prime} q_{j}^{\prime}=p_{i}^{\prime} p_{j}^{\prime}$, except that $\cos \left(q_{1}^{\prime} q_{2}^{\prime} / r\right)=-a$, and $3 a^{2}+b^{2}=1, a \neq b$.

Passing to the elliptic space $E_{8, r}$ yields two quintuples $p_{1}, p_{2}, \cdots, p_{5}$ and $q_{1}, q_{2}, \cdots, q_{5}$ such that the congruence $p_{1}, p_{2}, \cdots, p_{5} \approx q_{1}, q_{2}, \cdots, q_{5}$ cannot be extended to the whole space (that is, the two quintuples are not $f$-superposable) while, on the other hand, the congruence

$$
p_{1}, p_{2}, p_{3}, p_{4}, p_{5} \approx q_{1}, q_{4}, q_{5}, q_{2}, q_{8}
$$

can be extended to the whole $E_{3, r}$.

Thus the two quintuples are superposable, but the particular congruence $f$ between the sets cannot be extended to the whole space. Since, moreover, each two $f$-corresponding triples are easily seen to be $f$-superposable, it follows that the $g$-superposable quintuples are pseudo $f$-superposable.

The same example gives some information about another matter. While there obviously exist pseudo $f$-superposable sets of degree 1 with arbitrary power not exceeding $c$, and the existence of such sets of degree 2 follows from Theorem 3.3, there was some basis for the conjecture that if two subsets of $E_{8, r}$ are pseudo $f$-superposable of degree 3 , and neither set contains a planar $E_{2, r}$ quadruple, then each set consists of exactly four points. Examination of the two pseudo $f$-superposable quintuples exhibited above shows that neither contains a planar quadruple, and hence this conjecture is negated $\left({ }^{18}\right)$. Since, however, the two quintuples are actually superposable, it might be asked whether the $E_{k, r}(k>2)$ contains two non-superposable, pseudo $f$-superposable subsets of arbitrary power not exceeding $c$, when neither set has a $(k+1)$ tuple in $E_{k-1, r .}$. The existence of such sets has not yet been determined.

\section{BiBLIOGRAPHY}

1. G. Birkhoff, Metric foundations of geometry. I, Trans. Amer. Math. Soc. vol. 55 (1944) pp. $465-492$.

2. L. M. Blumenthal, Metric characterization of elliptic space, Trans. Amer. Math. Soc. vol. 59 (1946) pp. 381-400.

3. - Some imbedding theorems and characterization problems of distance geometry, Bull. Amer. Math. Soc. vol. 49 (1943) pp. 321-338.

4. D. van Dantzig and B. L. van der Waerden, Über metrisch homogene Rüume, Abh. Math. Sem. Hamburgischen Univ. vol. 6 (1928) pp. 374-376.

(13) The example was constructed by my student L. M. Kelly for this purpose.

UNIVERSITY OF MisSOURI, Columbia, Mo. 